\title{
Chandra Multiwavelength Project (ChaMP): Normal Galaxies at Intermediate Redshift
}

\author{
D.-W. Kim ${ }^{1}$, W. A. Barkhouse ${ }^{1}$, P. J. Green ${ }^{1}$, E. R. Colmenero ${ }^{2}$, \\ M. Kim ${ }^{1}$, A. Mossman ${ }^{1}$, E. Schlegel ${ }^{1}$, J. D. Silverman ${ }^{1}$, T. Aldcroft ${ }^{1}$, \\ C. Anderson ${ }^{1}$, H. Tananbaum ${ }^{1}$, and B. J. Wilkes ${ }^{1}$ \\ ${ }^{1}$ Smithsonian Astrophysical Observatory, 60 Garden Street, Cambridge, MA 02138, USA \\ email: kim@cfa.harvard.edu \\ ${ }^{2}$ South African Astronomical Observatory
}

\begin{abstract}
We have investigated 136 Chandra extragalactic sources without broad emission lines, including 93 NELG and 43 ALG. Based on $f_{X} / f_{O}, L_{X}$, X-ray spectral hardness and optical emission line diagnostics, we have conservatively classified 36 normal galaxies $(20$ spirals and 16 ellipticals) and 71 AGNs. Their redshift ranges from 0.01 to 1.2, while normal galaxies are at $\mathrm{z}=0.01-0.3$. Our sample galaxies appear to share similar characteristics with local galaxies in terms of X-ray luminosities and spectral properties, as expected from the X-ray binary populations and the hot ISM. In conjunction with normal galaxies found in other surveys, we found no statistically significant evolution in $L_{X} / L_{B}$, within the limited $\mathrm{z}$ range $(<0.1)$. We have built our $\log (\mathrm{N})-\log (\mathrm{S})$ relationship of normal galaxies in the flux range, $f_{X} \quad(0.5-8.0)=10^{-15}$ $10^{-13} \mathrm{erg} \mathrm{sec}^{-1} \mathrm{~cm}^{-2}$, after correcting completeness by a series of simulations. The best-fit slope is -1.5 for both soft $(0.5-2.0 \mathrm{KeV})$ and broad $(0.5-8.0 \mathrm{KeV})$ energy bands, which is considerably steeper than that of AGN-dominated cosmic background sources at faint fluxes, but slightly flatter than the previous estimate, indicating normal galaxies will exceed in number over the AGN population at $f_{X}<10^{-18} \mathrm{erg} \mathrm{sec}^{-1} \mathrm{~cm}^{-2}$.
\end{abstract}

Keywords. surveys - X-rays: galaxies - X-rays: general.

\section{Introduction}

To understand the formation and evolution of galaxies, it is important to study galaxies at a wide range of redshifts from the time when they form and rapidly evolve. The Chandra multiwavelength project (ChaMP), with the advantage of a wide area coverage, allow us to investigate a well-defined galaxy sample at redshifts intermediate between distant galaxies found in the Chandra Deep Field (CDF) and the local galaxies. In this paper, we will utilize the ChaMP sample in conjunction with normal galaxies from the deep surveys and apply X-ray properties obtained from well-studied local galaxies to address the classification, X-ray luminosity evolution and number density distribution of normal galaxies.

\section{Classification}

Our sample consists of narrow emission line galaxies (NELG) and absorption line galaxies (ALG). Broad line AGNs/QSOs (with a line width $>1000 \mathrm{~km} \mathrm{~s}^{-1}$ ) and galactic stars are excluded. To further separate even the obscured AGNs (Type 2 or XBONGs) from normal galaxies, we apply several diagnostics: the X-ray luminosity, X-ray-to-optical flux ratio, X-ray spectral hardness and optical line ratios. We find that $f_{X} / f_{O}$ is the most efficient way to distinguish normal galaxies and AGNs when applied conservatively, i.e., normal galaxy if $f_{X} / f_{O}<0.01$ and AGN if $f_{X} / f_{O}>0.1$ (see Figure 1). We define 

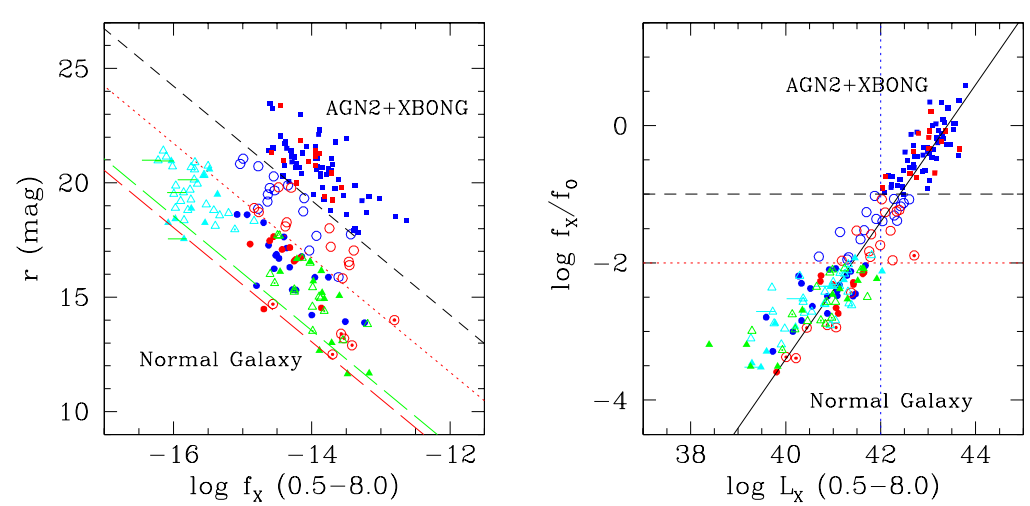

Figure 1. (a) The optical $r$ magnitude against $f_{X}$ and (b) the X-ray-to-optical flux ratio $\left(f_{X} / f_{O}\right)$ against $L_{X}$. NELGs and ALGs are distinguished by blue and red colors, respectively. Normal galaxies (with $f_{X} / f_{O}<0.01$ ) and AGNs (with $f_{X} / f_{O}>0.1$ ) are marked by filled circles and filled squares, respectively. Open circles mark those unclassified objects with intermediate $f_{X} / f_{O}$. Also plotted are normal galaxies identified in CDF-N (Hornschemeier et al. 2003) and XMM-Newton NHS (Georgantopoulos et al. 2005), marked by cyan and green triangles, respectively. The black dashed line and the red dotted line indicate $f_{X} / f_{O}=0.1$ and 0.01 , respectively. The diagonal solid line in Figure (b) indicates the best-fit linear relation for objects with $f_{X} / f_{O}$ $>0.01$.

$f_{X} / f_{O}$ by $f_{X}$ at $0.5-8.0 \mathrm{keV}$ and $f_{O}$ at the $\mathrm{r}$ band (e.g., Green et al. 2004). We note that in most cases classification by $f_{X} / f_{O}$ also satisfies other selection criteria. For example, all galaxies with $f_{X} / f_{O}<0.01$ are less luminous than $L_{X}=10^{42} \mathrm{erg} \mathrm{sec}^{-1}$ and all AGNs with $f_{X} / f_{O}>0.1$ are more luminous than $L_{X}=10^{42} \mathrm{erg} \mathrm{sec}^{-1}$ (see Figure 1b). However, with our conservative classification scheme, we can not classify objects with intermediate $f_{X} / f_{O}$. Their X-ray spectral hardness and optical line ratios indicate that these intermediate objects indeed consist of mixed types of AGNs and galaxies.

\section{Normal Galaxies}

In Figure 1, we also plot normal galaxies identified from the Chandra Deep FieldNorth (CDF-N; Hornschemeier et al. 2003) and the XMM-Newton Needle in the Haystack Survey (NHS; Georgantopoulos et al. 2005). Our sample consists of galaxies at redshifts $\mathrm{z}=0.01-0.3$, intermediate between the $\operatorname{CDF}(\mathrm{z}=0.1-1.0)$ and nearby local galaxies, and covers an intermediate flux range. Our sample is similar to the NHS $(z=0.005-0.2)$, but extends to a slightly higher $\mathrm{z}$ and a fainter X-ray flux. Combining all three samples allows us to cover a wide parameter space and to increase the statistical confidence. While galaxies from three different samples are distinct in their X-ray flux ranges due to the different observation depths, they are well mixed in the $f_{X} / f_{O}-L_{X}$ space (or in the $L_{O}-L_{X}$ space) with $L_{X}=10^{38}-10^{42} \mathrm{erg} \mathrm{sec}^{-1}$ and $\log f_{X} / f_{O}=-3.5-2$ (Figure 1 ).

While we impose a maximum $f_{X} / f_{O}\left(\log f_{X} / f_{O}=-2\right)$, there is no lower limit imposed in our selection. Therefore, this minimum $f_{X} / f_{O}$ must be real for normal galaxies and appears to remain the same in all three samples. It is interesting to note that the observed minimum $f_{X} / f_{O}$ is consistent with the lower limit of $L_{X}-L_{B}$ relationship of early type galaxies (red dashed line; Kim and Fabbiano 2004) and of spirals (green dashed 

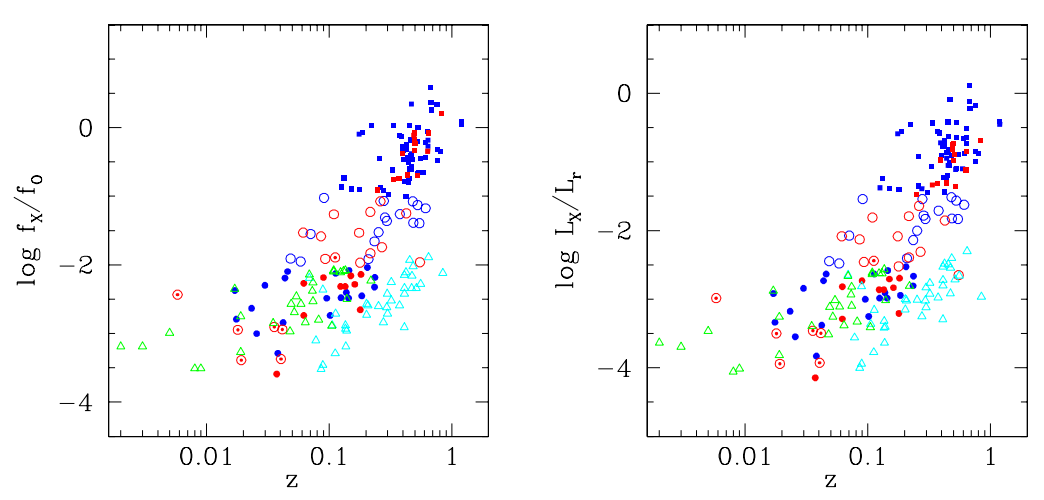

Figure 2. X-ray to optical flux/luminosity ratios as a function of $\mathrm{z}$.

line; Colbert et al. 2003). Those galaxies with the minimum $f_{X} / f_{O}$ represent X-ray faint (LMXB-dominant, gas-poor) early type galaxies, or quiescent (non-starburst) spiral galaxies. This indicates that the X-ray emission from X-ray binary populations in our $\mathrm{X}$-ray selected galaxies is similar to that expected from local normal galaxies.

\section{4. $L_{X}-L_{B}$ evolution}

As the star formation rate peaks at $\mathrm{z}=2-3$, the fossil record of past star formation imprinted on X-ray binaries could be detected by observing galaxies at high redshifts (e.g., Ghosh and White 2001; Ghosh in this conference). The X-ray luminosity could be 10-100 times higher than that in the local galaxies with HMXB peaking at $\mathrm{z}=1-2$ while LMXB at $\mathrm{z}=0.5-1.0$. We note that the average quantity of $f_{X} / f_{O}$ or $L_{X} / L_{O}$ as a function of $\mathrm{z}$ (as often used in the literature) could be seriously affected by selection effects (e.g., the upper limit of $f_{X} / f_{O}$ ) and/or AGN contamination. Instead, we test the X-ray luminosity evolution using the minimum of $f_{X} / f_{O}$ and $L_{X} / L_{O}$ as a function of z (as described in the previous section).

In Figure 2, we plot the X-ray-to-optical flux/luminosity ratios as a function of $\mathrm{z}$. Again we have plotted normal galaxies from the CDF-N and NHS surveys as well. The difference between flux and luminosity ratio is caused by the K-correction and the evolution correction. The combined sample is complete up to $\mathrm{z}=0.1$ and becomes incomplete at higher $\mathrm{z}$ due to the flux limit. Within this limited $\mathrm{z}$ range $(\mathrm{z}<0.1$ or look back time of $\sim 1 \mathrm{Gyr}$ ), the minimum value of $L_{X} / L_{r}$ (or $f_{X} / f_{O}$ ) remains constant, indicating that no significant change in the X-ray properties of normal galaxies, hence no luminosity evolution in the $\mathrm{X}$-ray binary population up to $\mathrm{z}=0.1$. It is critical to include galaxies at higher $\mathrm{z}(\mathrm{z}=0.5-1.0)$, but that will require the next generation $\mathrm{X}$-ray mission such as Gen X.

\section{Normal galaxy Log N- LogS relation}

In Figure 3, we plot the $\log (\mathrm{N})-\log (\mathrm{S})$ relation of the ChaMP normal galaxies. Our $\log (\mathrm{N})-\log (\mathrm{S})$ relations in the soft $(0.5-2.0 \mathrm{keV})$ and broad $(0.5-8.0 \mathrm{keV})$ bands appear 


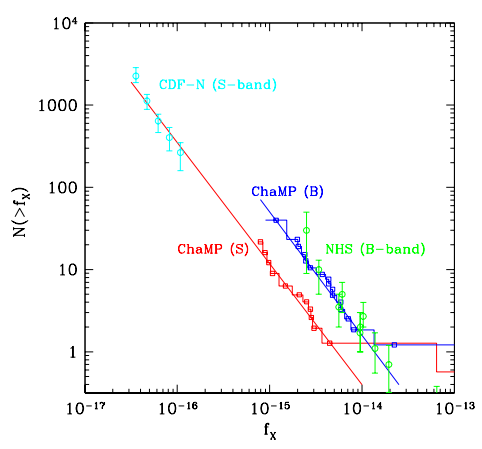

Figure 3. $\log \mathrm{N}-\log \mathrm{S}$ relation of normal galaxies.

to be in the same shape, with the Euclidean slope of $-1.5 \pm 0.15$ (the best-fit powerlow distribution is plotted in the figure.) This is not surprising, because we are dealing with a homogeneous sample of galaxies with a relatively small amount of obscuration. This is in contrast to AGN-dominated cosmic background sources, where obscured and unobscured X-ray sources contribute differently in different energy bands (eg., Kim et al. 2004). We also note that unlike the broken power-law distribution of cosmic background AGNs with a break at $f_{X}(0.5-2.0 \mathrm{keV})=6 \times 10^{-15} \mathrm{erg} \mathrm{cm}^{-2} \mathrm{~s}^{-1}$ (e.g., Kim et al. 2004), the $\log (\mathrm{N})-\log (\mathrm{S})$ of normal galaxies is well reproduced by a single power-law in a wide range of $f_{X}$ (see also Tajer et al. 2005). Also plotted in Figure 3 are those previously determined with the CDF sample in the S-band (Hornschemeier et al. 2003) and the NHS sample in the B-band (Georgakakis et al. 2004). It appears that our S-band $\log (\mathrm{N})-\log (\mathrm{S})$ can be connected to that of the CDF, if extrapolated. However, the CDF data point at the faintest $f_{X}\left(\sim 3 \times 10^{-17} \mathrm{erg} \mathrm{s}^{-1} \mathrm{~cm}^{-2}\right)$ is slightly higher than the extrapolated value and their best-fit slope, -1.74 , is slightly steeper than ours. Our B-band $\log (\mathrm{N})-\log (\mathrm{S})$ is statistically identical with that of the NHS, except our data extend to lower fluxes with smaller error bars. Since the $\log (\mathrm{N})-\log (\mathrm{S})$ relation of normal galaxies is considerably steeper than that of AGN-dominated cosmic background sources (with a slope of 0.60.7 ), we determine that normal galaxies will exceed in number over the AGN population at $f_{X}<10^{-18} \mathrm{erg} \mathrm{sec}-1 \mathrm{~cm}^{-2}$. which is slightly fainter than previous estimates by Hornschemeier et al. (2003) and Bauer et al. (2004).

\section{References}

Bauer, F. E., et al. 2004, astro-ph/0408001

Colbert, E. J. M., et al. 2003, ApJ, 602, 231

Georgakakis, A. E., Georgantopoulos, I., Basilakos, S., \& Plionis, M. 2004, astro-ph/0407387

Georgantopoulos, I., Georgakakis, A., \& Koulouridis, E. 2005, astro-ph/0503494

Ghosh, P. \& White, N. E. 2001, ApJ, 559, L97

Green et al. 2004, ApJS, 150, 43

Hornschemeier, A. E., et al. 2003, ApJ, 126, 575

Kim, D.-W. \& Fabbiano 2004, ApJ, 611, 846

Kim, D.-W., et al. 2004a, ApJS, 150, 19

Kim, D.-W., et al. 2004b, ApJ, 600, 59

Maccacaro, T., et al. 1988, ApJ, 326, 680

Tajer, M., Trinchieri, G., et al. 2005, AA, submitted (also astro-ph/0412588) 